\title{
Numerical Evaluation of the Surface Energy of Polyhedral Nanoparticles
}

\author{
Takuya Uehara1 ${ }^{*}$, Junya Fujiwara ${ }^{2}$ \\ ${ }^{1}$ Department of Mechanical Systems Engineering, Yamagata University, Yonezawa, Japan \\ ${ }^{2}$ Graduate School of Science and Engineering, Yamagata University, Yonezawa, Japan \\ Email: *uehara@yz.yamagata-u.ac.jp
}

How to cite this paper: Uehara, T. and Fujiwara, J. (2020) Numerical Evaluation of the Surface Energy of Polyhedral Nanoparticles. Materials Sciences and Applications, 11, 837-850.

https://doi.org/10.4236/msa.2020.1112055

Received: November 9, 2020

Accepted: December 14, 2020

Published: December 17, 2020

Copyright () 2020 by author(s) and Scientific Research Publishing Inc. This work is licensed under the Creative Commons Attribution International License (CC BY 4.0).

http://creativecommons.org/licenses/by/4.0/ (c) (i) Open Access

\begin{abstract}
Nanoparticles have been used widely in various fields, and their size and shape greatly affect the functional properties. Therefore, controlling the morphology of the particles is important, and evaluation of the surface energy is indispensable for that purpose. In this study, the surface energy of nanoparticles was evaluated by numerical simulation and formulated in a polynomial equation. First, molecular dynamics simulations were carried out for variously shaped polyhedral nanoparticles. A cube and an octahedron were introduced as reference shapes, and truncated hexahedrons and truncated octahedrons were created by cutting out their vertices. The surface energy was plotted for various polyhedrons. The lowest energy was observed in an octahedron because of the stability of the (111) plane, and the highest energy was observed in a cube because of the relatively higher energy of the (100) plane. Then, the surface energy was formulated in a polynomial equation, in which the parameters obtained by the molecular-dynamics simulations were introduced. As a result, stability of the octahedron and relative instability of the cube were fairly captured by the proposed polynomial equation, while a slight underestimation was inevitable. Finally, the parameters were revised to continuous numbers to extend the application range. Consequently, an application for various materials, such as a cube having equivalent stability to an octahedron, was demonstrated by imposing rather exaggerated parameters.
\end{abstract}

\section{Keywords}

Surface Energy, Nanoparticle, Fcc Crystal, Interatomic Potential, Molecular Dynamics, Computer Simulation 


\section{Introduction}

A small particle with a nano-meter order diameter is called a "nanoparticle", and it has been used in a wide range of fields such as electronics, photonics, environmental engineering, medical sciences, and pharmaceuticals [1] [2]. In practical application, nanoparticles are typically scattered in a base material rather than being used as a single particle. Nonetheless, the individual size and shape of every particle affect the properties of the entire material. Therefore, controlling the morphology of nanoparticles is a key issue in practical application, and theoretical understanding of the morphological stability in an atomistic scale is indispensable. There are several ways of producing nanoparticles. These are typically divided into physical and chemical processes. In the latter, the chemical reaction should be considered, and the covalent bonding and electronic structure should be modeled based on the first-principle or quantum chemical analyses. In contrast, the mechanism in the physical process, which is dominant for metallic particles, is rather simple because homogeneous interatomic potentials can be applied to an atomistic-order modeling. The molecular dynamics (MD) method [3], by which the motions of all atoms in the considered system are tracked, is suitable for simulating the formation process of nanoparticles and evaluating their stability. The parameters in the interatomic potential, however, depend on the materials, and a wide variety of stable shapes can be observed depending on the parameters. Hence, the method is suitable for studying a specified material but ineffective for general investigation. Meanwhile, an approximation of the nanoparticles based on polyhedral shape is important and effective for estimating the stability of particles and capturing their characteristics. In this regard, various studies have been conducted [4]-[9]. However, a general theory on the stability of nanoparticles remains incomplete.

Concerning the evaluation of surface energy, a phase-field method [10] [11] is another potential approach. In this method, optimization of various types of morphology, including the microstructure of materials and mechanical structure of beams, is possible based on the notion of energy minimization. Therefore, if the surface energy is formulated using continuous variables, it would be possible to predict the stable shape of the particles by applying the energy formula to the phase-field method. The author demonstrated phase-field simulation for evaluating the stability of polyhedral shapes in foam materials and for investigating domain tessellation in three-dimensional space [12] [13]. Currently, we are motivated to apply this procedure in evaluating the stability of nanoparticles. As a first step, in this paper, an expression of the surface energy in a polynomial equation is proposed following MD simulations to obtain relevant parameters. Representative polyhedrons are considered in a series of truncated hexahedrons and truncated octahedrons by cutting the vertices of a cube or an octahedron [14]. In addition to the parameters obtained by MD simulations, several trials are conducted to indicate the possibility of application to various materials. 


\section{Making Polyhedron Models}

\subsection{Target Polyhedrons}

Face-centered-cubic (fcc) metals are considered in this study. In the fcc crystal, (111) planes are the most stable, and this is followed by (100) planes, and other planes are relatively unstable. As such, only these two planes were focused on in this study. One of the typical polyhedral shapes is a cube, which has six (100) planes, 12 edges, and eight vertices. Another preferable shape is an octahedron, which comprises eight (111) planes, 12 edges, and six vertices. By setting these two shape types as the standard shapes for the current study, various polyhedrons were created as shown in Figure 1. Truncated hexahedrons were created by cutting the eight vertices of a cube in Figure 1(a), generating six octagons of the (100) planes and eight triangles of the (111) planes as shown in Figure 1(b). Conversely, truncated octahedrons were created by cutting the vertices of an octahedron shown in Figure 1(f), which comprised eight regular hexagons and six

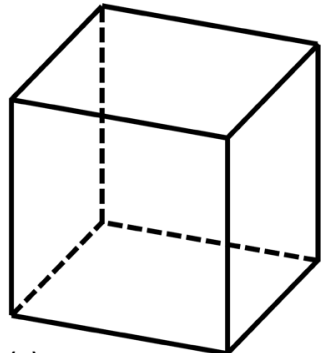

(a)

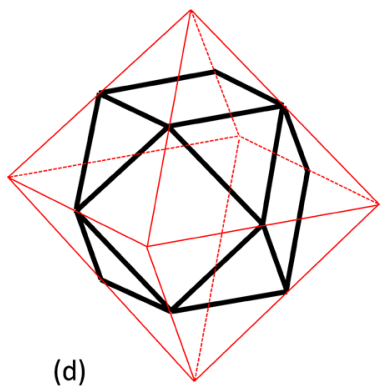

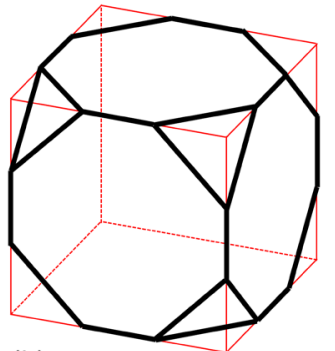

(b)

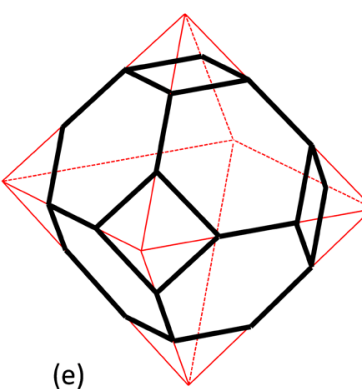

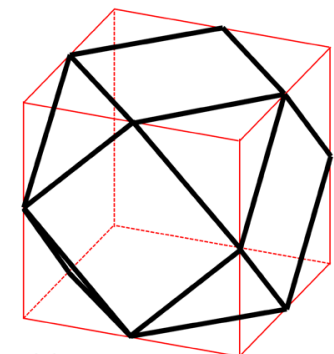

(c)

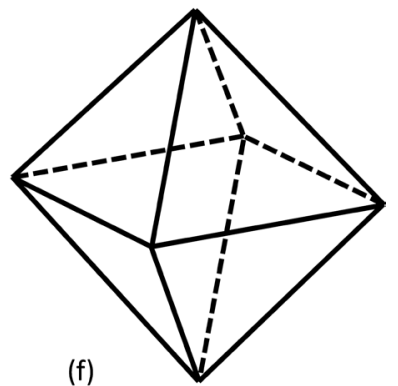

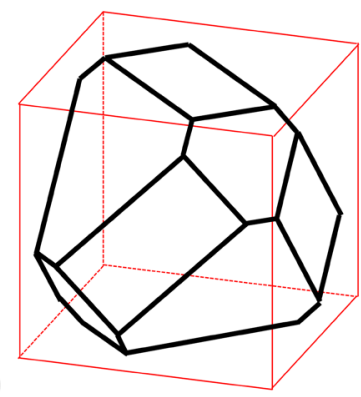

(h)

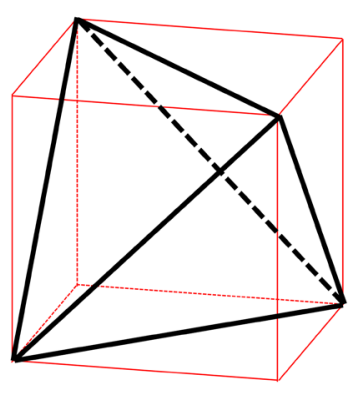

(g)

Figure 1. Target polyhedrons: a truncated hexahedron (b) and truncated octahedron (e) are created by cutting the vertices of a cube (a) and regular octahedron (f), respectively. Cuboctahedrons (c) and (d) are created by either from cube or octahedron, respectively. In addition to these polyhedrons, a regular tetrahedron $(\mathrm{g})$ and an asymmetric truncated octahedron (h) are considered. 
squares as shown in Figure 1(e). Generally, the hexagons have three long edges and three short edges. When the lengths of all edges become the same, the polyhedron is called a Kelvin cell. This shape is geometrically special in the sense that it is the best space-filling shape in three-dimensional space with the smallest surface area under a given volume. A cuboctahedron was created in both ways by cutting the cube and by cutting the octahedron, as shown in Figure 1(c) and Figure 1(d), respectively.

All these polyhedrons comprise either or both the (100) and (111) planes. In addition to these shapes, a regular tetrahedron and an asymmetric truncated octahedron illustrated in Figure 1(g) and Figure 1(h), respectively, were considered. All four faces of a regular tetrahedron are the (111) plane, and six edges are included. The number of faces, edges and vertices of an asymmetric truncated octahedron is the same as those of a truncated octahedron, while the faces differently comprise four large hexagons, four small hexagons, and six rectangles. Many other shapes can be generated using the (100) and (111) planes, but only the shapes mentioned above were targeted herein because of their symmetric character.

In these polyhedrons, cube, regular octahedron, and regular tetrahedron shapes are termed regular polyhedrons or Platonic solids, in which all faces are identical regular polygons, and all edges and vertices have the same characteristics. In addition, a cuboctahedron and Kelvin cell are termed semi-regular polygons or Archimedean solids. The number of faces, edges, and vertices as well as the Miller indexes of the relevant faces are listed in Table 1, in which the abbreviated designations used in this paper are also noted.

\subsection{Parameters Determining the Polyhedrons}

To parameterize the size and shape of the polyhedrons, the following variables were introduced: $l_{\mathcal{O}}$ the edge length of the original cube; $c$, the cutting length from the vertex of the cube along the edge; $l_{o}$, the edge length of the original octahedron; and $d$, the cutting length from the vertex of the octahedron along the edge. The ranges of $c$ and $d$ are limited as $0 \leq c \leq l_{c} / 2$, and $0 \leq d \leq l_{o} / 2$, respectively. The cuboctahedron is generated from the cube when $c=1 / 2$ and from the octahedron when $d=I_{o} / 2$. The Kelvin cell is formed from the octahedron when $d=I_{d} / 3$. Here, the truncated octahedrons can be generated from a cube by setting the value of $c$ as $l_{c} / 2 \leq c \leq l_{c}$. However, this process induces the overlap of the cut parts, which hinders intuitive understanding. Nonetheless, it is useful for simplifying the parameters. Therefore, in this paper, the parameters $I_{c}$ and $c$ are used to identify the shape and size of a polyhedron. The cutting lengths from alternative vertices are set as $c_{1}$ and $c_{2}$ (the diagonal vertices in the original square face have the same index) under a limitation of $l_{c} \leq\left(c_{1}+c_{2}\right) \leq 2 l_{c}$. Finally, the size of a regular tetrahedron is simply expressed by the edge length, $l_{t}$.

The length, $l$, irrespective of the subscript, is expressed by the number of atoms along the edge and is denoted by integer $n$. Cutting parameter $c$ is also 
Table 1. Number of faces, edges, and vertices of the polyhedrons targeted in this paper.

\begin{tabular}{|c|c|c|c|c|}
\hline Name & Abbreviation & Faces & Edges & Vertices \\
\hline Cube & Cube & $6(100)$ & $12(100)-(100)$ & $8(100)-(100)-(100)$ \\
\hline Truncated hexahedron & trHexa & $\begin{array}{l}6(100) \\
8(111)\end{array}$ & $\begin{array}{l}12(100)-(100) \\
24(111)-(100)\end{array}$ & $24(100)-(100)-(111)$ \\
\hline Cuboctahedron & Cubocta & $\begin{array}{l}6(100) \\
8(111)\end{array}$ & $24(111)-(100)$ & $12(100)-(100)-(111)-(111)$ \\
\hline Truncated octahedron & trOcta & $\begin{array}{l}6(100) \\
8(111)\end{array}$ & $\begin{array}{l}24(111)-(100) \\
12(111)-(111)\end{array}$ & $24(100)-(111)-(111)$ \\
\hline Octahedron & Octa & $8(111)$ & $12(111)-(111)$ & $6(111)-(111)-(111)-(111)$ \\
\hline Tetrahedron & Tetra & $4(111)$ & $6(111)-(111)^{\prime}$ & $4(111)-(111)-(111)$ \\
\hline $\begin{array}{l}\text { Asymmetric truncated } \\
\text { octahedron }\end{array}$ & astrOcta & $\begin{array}{l}6(100) \\
8(111)\end{array}$ & $\begin{array}{l}24(111)-(100) \\
12(111)-(111)\end{array}$ & $24(100)-(111)-(111)$ \\
\hline
\end{tabular}

expressed by the integer number of atomic layers. The models are identified by the abbreviated notation for their shape, as listed in Table 1 , and by the values of $n$ and $c$. For example, "Cube (n12)" refers to the cube with $I_{c}=12 L_{a}$ ( $L_{a}$ : lattice constant), and "trOcta (n12c6)" denotes the truncated octahedron made by cutting six atomic layers from the vertices of Cube (n12).

\section{Surface-Energy Evaluation by MD Simulation}

\subsection{Simulation Method}

The surface energy of nanoparticles can be directly calculated using an atomistic model assuming a specific interatomic potential function. In this study, the Lennard-Jones potential function was applied. A quantitative evaluation of a specific material is sensitive to the choice of potential function, but we applied a simple two-body function because our purpose was to present a general methodology. The atoms were arranged on the lattice points of the fcc structure in a cubic space so that the (100), (010), and (001) planes are on the $y-z, z-x$ and $x-y$ planes, respectively. Then, an arbitrary polyhedral shape was formed by removing the atoms out of the target range. For the truncated octahedron, a regular octahedron was made first by the above-mentioned procedure, and then, the atoms in the cutting area were deleted. The initial position of the atoms was equilibrated by the MD method for the 10,000 time-steps, and the surface energy and other properties were calculated. The fundamental equations involved are as follows.

Newton's equation of motion:

$$
m_{i} \ddot{r}_{i}=\boldsymbol{F}_{i}=-\sum_{j \neq i} \frac{\mathrm{d} \phi_{i j}}{\mathrm{~d} r} \frac{\boldsymbol{r}_{i j}}{r_{i j}} .
$$

Interatomic potential function (Lennard-Jones type):

$$
\phi_{i j}=4 \varepsilon\left(\left(\frac{\sigma}{r_{i j}}\right)^{12}-\left(\frac{\sigma}{r_{i j}}\right)^{6}\right) .
$$

Here, $m_{i}$ is mass of the $i$-th atom, $\boldsymbol{r}_{i}$ is the position vector of the $i$-th atom, and 
$F_{i}$ is the force acting on the $i$-th atom, which is defined as the position derivative of interatomic potential function $\phi_{i j}$ The parameters $\varepsilon$ and $\sigma$ depend on the material, and a nondimensional analysis is demonstrated by standardizing the energy and length based on $\varepsilon$ and $\sigma$, respectively.

Common-neighbor analysis (CNA) [15] is applied to determine the crystal structure. Furthermore, whether an atom is in the bulk, on a surface, on an edge, or a vertex can be specified by this method. The structures considered in this paper are listed in Table 2, where the color column indicates the legend used in the presentation of the results.

\subsection{Definition of the Surface Energy of an Atomic Model}

Surface energy $\gamma$ is defined as the increase in energy due to the existence of a surface and is expressed as the difference in the energy between the atoms on a surface and the atoms in bulk where the energy is unaffected by the surface. Here, the value of $\gamma$ is calculated as the energy per atom in the atomistic model, while surface energy in general is typically defined as a value per area. In a particle model, if the atoms are observably divided into surface and bulk atoms, the surface energy can be calculated as follow:

$$
\gamma=\frac{\sum_{i \in \text { surface }}\left(e_{i}^{s}-e^{b}\right)}{S},
$$

where $e_{i}^{s}$ and $e^{b}$ are the energy of the atoms on surface and in bulk, respectively, and $S$ is the surface area. However, establishing a definition of the surface atom is difficult because the influence of the surface acts across more than one atomic layer. Now, assuming the internal atoms are unaffected by the surface and have the same energy as $e^{b}$, Equation (3) can be calculated as follows:

$$
\gamma=\frac{\sum_{i \in \text { particle }}\left(e_{i}-e^{b}\right)}{S}
$$

where the summation in taken over all atoms in the model without the need for distinguishing the surface and bulk atoms, and the surface energy finally corresponds to the difference in energy between the particle model and bulk model [16]. The bulk energy $e^{b}$ is obtained by a separate simulation using a fully bulk model with periodic boundary condition.

Another difficulty is defining the surface area of the particle. In this study, the particle model was created based on a polyhedral shape, and the surface area of

Table 2. Surfaces and edges determined by CNA and color legend used in this paper.

\begin{tabular}{cccc|cccc}
\hline No. & Color & Type & Property & No. & Color & Type & Property \\
\hline 1 & $\bigcirc$ & bulk & fcc & 5 & $\bigcirc$ & edge & $(100)-(111)$ \\
2 & - & bulk & hcp & 6 & & edge & $(111)-(111)$ \\
3 & - & surface & $(111)$ & 7 & $\bigcirc$ & edge & $(100)-(100)$ \\
4 & & surface & $(100)$ & 8 & $\bigcirc$ & others & \\
\hline
\end{tabular}


the initial polyhedron was applied by neglecting a slight volume change and roughing of the surface occurred during relaxation calculation.

\subsection{Results of MD Simulation}

Snapshots of the MD models after the relaxation steps are shown in Figure 2, where a truncated hexahedron (a), a cuboctahedron (b), and a truncated octahedron (c) are shown, and the color indicates CNA distinction (i) and potential energy (ii). The polyhedral shapes were maintained via the relaxation steps for all models. In CNA colors, atoms on the surface and edges are properly distinguished, whereas some of the vertices on the edge are not specified. The bulk atoms, depicted in blue, are visible between the surface atoms. The potential energy of the bulk atoms is significantly lower than that of the surface atoms, and the atoms are colored in blue in Figures (ii). The face atoms have a higher energy compared to the bulk atoms, and both the (100) and (111) atoms are colored in green, since the difference in energy is relatively small in the exhibited range. The edge atoms have much higher energy than the face atoms. The edge between two (111) faces and the edge between (100) and (111) faces are shown in yellow, whereas the edge between two (100) faces is denoted in red, which represents that the (100)-(100) edge has the highest energy in the model. This is clear from the fact that the angle between the two faces is sharper compared with other edges.

Figure 3 shows the variations of the potential energy per atom averaged across all atoms (a) and the average of the surface atoms (b). The values fluctuated initially from the artificially given initial position but soon converged by

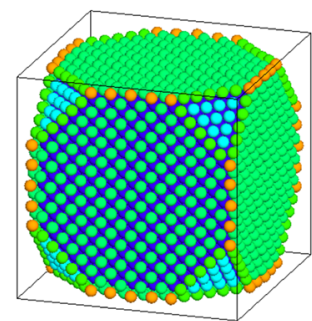

(i) $\mathrm{CNA}$

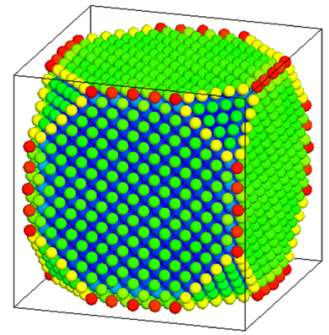

(ii) Potential energy

(a) Truncated hexahedron (n11c8)

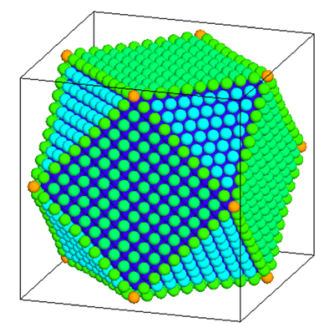

(i) $\mathrm{CNA}$

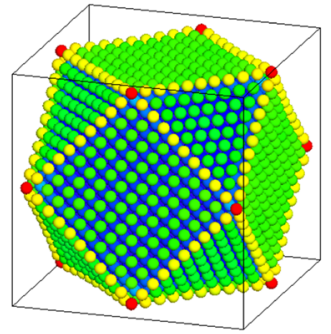

(ii) Potential energy

(b) Cuboctahedron

(n11)

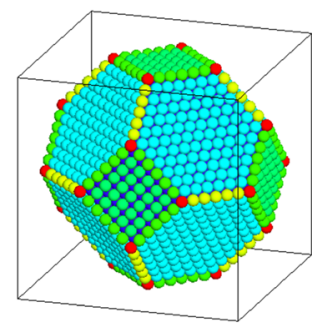

(i) $\mathrm{CNA}$

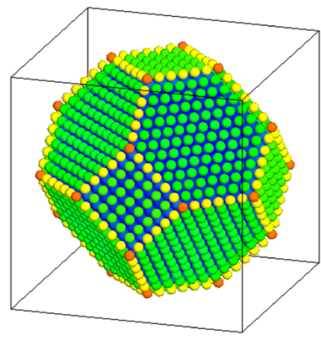

(ii) Potential energy

(c) Kelvin cell (n13)

Figure 2. MD model and simulation results; (a) truncated hexahedron with $n=11, c=8$, (b) cuboctahedron with $n=11$, and (c) truncated octahedron (Kelvin cell) with $n=13$, colored by CNA (i) and potential energy (ii). Color index for CNA is listed in Table 2. 
the $2000^{\text {th }}$ time-step. The energy of the entire particle was slightly low in the Kelvin cell, and the truncated hexahedron and cuboctahedron were almost the same as shown in Figure 3(a). However, if only the surface atoms are considered (as shown in Figure 3(b)), the cuboctahedron shows a lower energy than the truncated hexahedron, and the low energy of the Kelvin cell is more significant. This is because the stable (111) plane occupies a larger fraction in the Kelvin cell, and the presence of the (100)-(100) edges affect the high energy of the cubic shape.

Various polyhedron models were also created, and MD simulations were carried out. The configurations of the atoms are shown in Figure 4, where different colors indicate the potential energy. The (100)-(100) edges in the cube and truncated hexahedron had higher energies than the (100)-(111) and (111)-(111) edges. The (111)-(111) edges in the regular tetrahedron are different from the
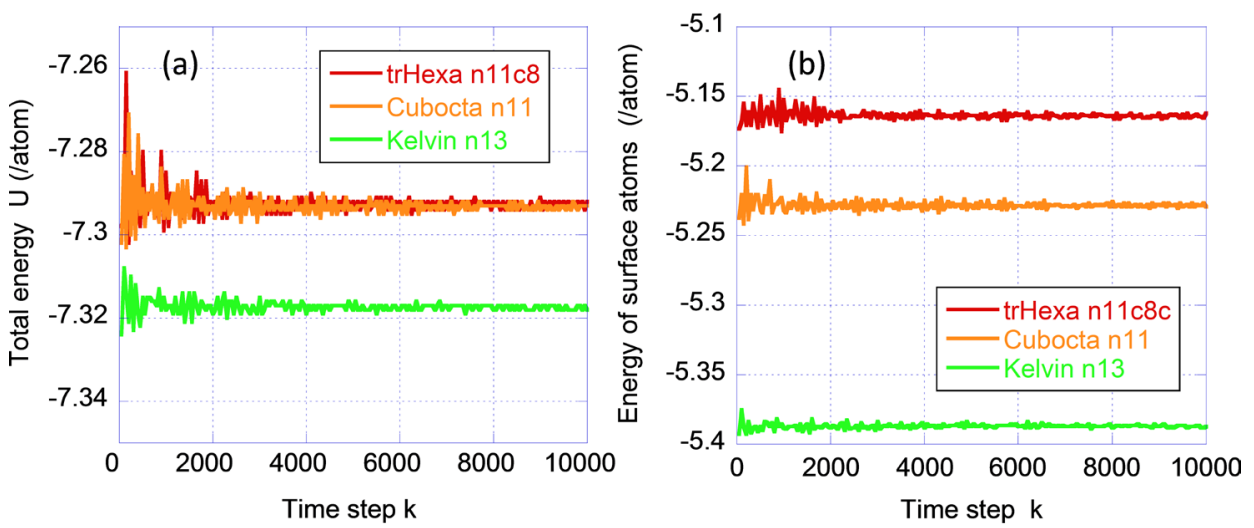

Figure 3. Variation of the potential energy of polyhedral particles: (a) total average of all atoms, (b) average of surface atoms (including face, edge and vertices).

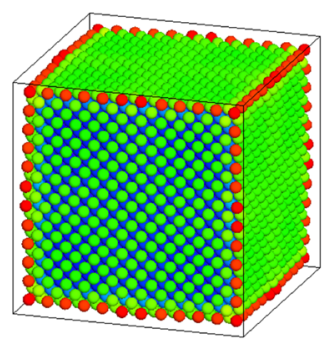

(a) Cube (n12)

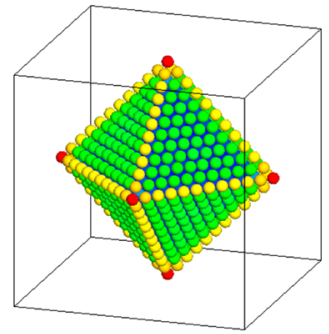

(d) Regular octahedron (n12)

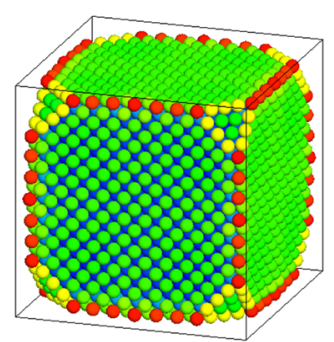

(b) Truncated hexahedron (n11c6)

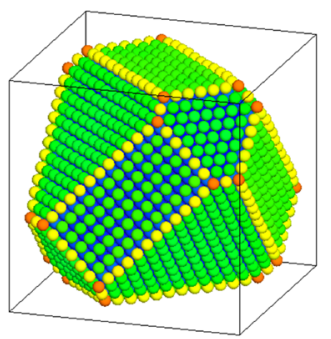

(e) Asymmetric tr. Octa. (n12)

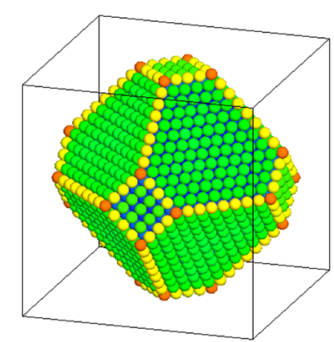

(c) Truncated octahedron (n13c22)

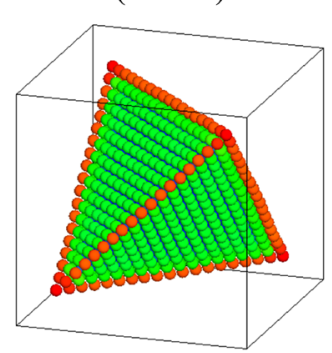

$-3.2$

(f) Regular tetrahedron (n12)

Figure 4. Simulation results for verious polyhedrons. The different colors indicate the potential energy. 
(111)-(111) edges in the octahedron; i.e., the angle between the faces in the tetrahedron is much sharper than that of the octahedron. The actual Miller indices are $(111)$ and $(\overline{1} 11)$ or $(1 \overline{1} 1)$ in the octahedron, and (111) and $(\overline{1} \overline{1} 1)$ in the tetrahedron.

The total energy averaged across all atoms in a particle, $U$, and the average of the atoms on the surface, $e$, are plotted in relation to the number of atoms in a particle, $N$, in Figure 5(a) and Figure 5(b), respectively. The total energy decreased as the particle became larger because the fraction of surface atoms with high energy against bulk atoms with low energy decreased. The difference in the shape of a particle did not particularly affect the value as shown in Figure 5(a). However, the energy of the surface atoms showed a notable difference (see Figure 5(b)). Cubes comprising only (100) faces had the highest energy, whereas tetrahedrons and octahedrons, both of which comprised (111) faces, had the lowest energy. This indicates that the energy of the (100) face is higher compared with that of the (111) face. Furthermore, face energy was dominant for the total surface energy. For other polyhedrons, the energy became lower as the (100) area decreased and the (111) increased; the energy decreases in the order of cube, truncated hexahedron, cuboctahedron, truncated octahedron, and octahedron. The difference between the tetrahedron and octahedron was their constituent edges. As shown in Figure 4(d) and Figure 4(f), the edge of the tetrahedron is sharper and has higher energy, but the total length of the edges is longer in the octahedron. These effects compensate for one another, and the resulting energy came close to an almost identical value.

\section{Formulation of Surface Energy}

\subsection{Polynomial Equation}

According to the MD simulations in the previous section, the surface energy $\gamma$ of nanoparticles is assumed to be approximated by the following equation:
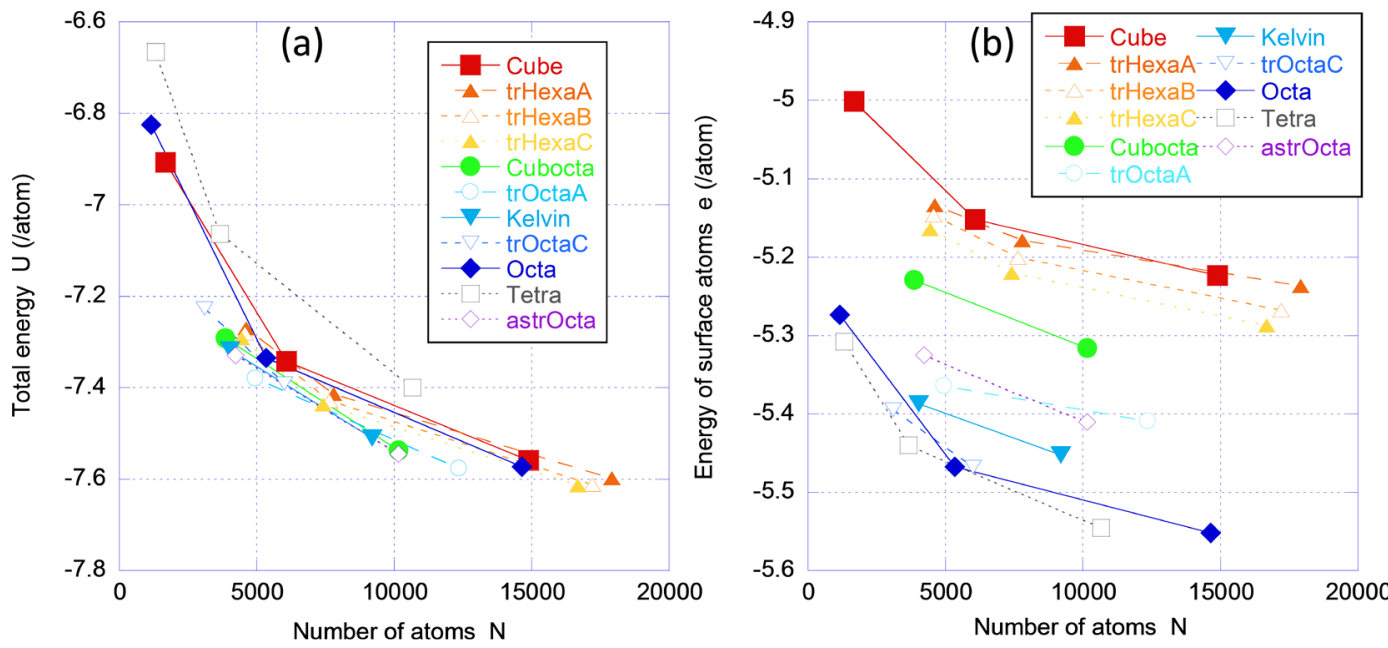

Figure 5. Potential energy vs. the number of atoms in various polyhedron models: (a) total average of all atoms; (b) average of surface atoms (including faces, edges and vertices). 


$$
\gamma=\frac{1}{S} \sum_{k \in D_{k}} N_{k}\left(e_{k}-e_{0}\right)
$$

Here, $D_{k}$ is the element constituting the surface, e.g., the (111) face and (100)-(111) edge, $e_{k}$ is the energy per atom depending on the element, $e_{0}$ is the average energy in the bulk region, $N_{k}$ is the number of atoms in $D_{k}$, and $S$ is the surface area. The values of $e_{k}$ were obtained by the MD simulations. For example, the value for $\{k$ : (111) face $\}$ was evaluated from the calculation for a regular octahedron (n28) by taking the average value of the (111) atoms in the model. The energy values varied depending on the shape and size of the MD models, even those with the same face type. Here, the values for the model having the largest area or longest edges were selected because these tend to converge as the size becomes larger. The values and relevant polyhedron models are listed in Table 3. Using Equation (5) and the parameters in Table 3, the surface energy of the cube, regular octahedron and arbitrary polyhedron produced by cutting their vertices were calculated.

\subsection{Fitting Results for MD}

The calculated surface energy is plotted in Figure 6. The values for the cubes

Table 3. Energy per atom on the specific elements and the polyhedron model from which the values were taken.

\begin{tabular}{ccc|ccc}
\hline Element & Value & Model & Element & Value & Model \\
\hline (111) face & -5.709 & octa n28 & vertex 111-111-111-111 & -2.377 & octa n28 \\
$(100)$ face & -5.352 & cube n16 & vertex 111-111-100-100 & -3.074 & cubocta n15 \\
$111-100$ edge & -4.462 & cubocta n15 & vertex 111-111-100 & -3.704 & Kelvin n17 \\
$111-111$ edge & -4.301 & trOcta n17c22 & vertex 111-100-100 & -3.300 & trHexa n17c22 \\
$100-100$ edge & -3.398 & trHexa n17c06 & vertex 100-100-100 & -2.107 & Cube n16 \\
\hline
\end{tabular}

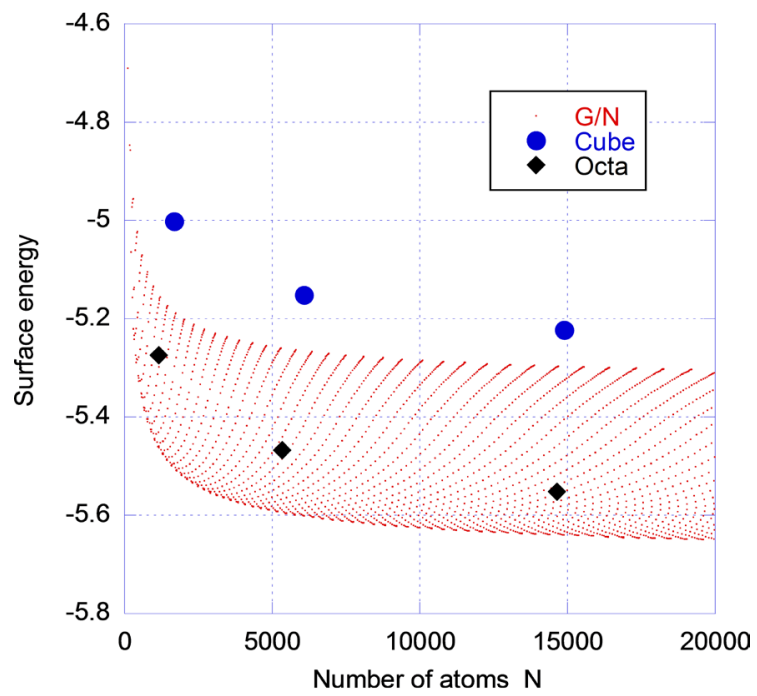

Figure 6. Surface energy fitted by polynomial equation based on the parameters obtained by the MD simulation. 
and octahedrons presented in Figure 5(b) are also plotted as a reference for the maximum and minimum range obtained by MD simulation. The calculated energy is distributed with a band-like area, and the upper and lower ends correspond to the results for the cube and octahedron, respectively. The overall tendency is appropriately presented, while the values are slightly lower than the MD results. The reason for this difference is that the increase in energy around the surface element is not included in Equation (5). The atoms in a few layers in the depth direction from the surface are affected and generally have higher energy. Additionally, even for the atoms in the (111) surface, the energies in the center and near the edge are slightly different. These effects should be introduced for precise evaluation. Nonetheless, this study concludes that the overall tendency was captured using this method. In the following section, the prediction of the surface energy of various nanoparticles is presented using virtual parameters.

\subsection{Modification to Continuous Parameter}

The calculation in the previous section was based on atomic arrangement, and the edge and cutting length were represented by integer numbers. For a more systematic investigation, the lengths were revised to continuous real numbers. Then, the cutting length from the cube vertex was standardized by the edge length $L$ of the original cube, i.e., $c=0$ is the cube, $c=0.5$ is the cuboctahedron, $c=0.67$ is the Kelvin cell, and $c=1$ is the regular octahedron.

The energy per atom was also modified to the energy per area for the faces, energy per length for the edges, and energy per point for the vertices. Corresponding values were obtained by considering the planar and linear density of each face or edge. This enabled the evaluation of the size dependency of the surface energy. Then, size parameters $l$ and $c$ for different shapes could be defined under a constant volume, by which we could compare the difference in shape of the surface energy under a constant-volume condition.

Figure 7 (a) shows the variation of the surface area and edge length under constant volume $V=500$. The surface area decreased as the cutting length increased in the range of $0<c<0.5$, which means that the maximum and minimum surface areas were obtained for the cube and cuboctahedron, respectively. It can be intuitively understood that the surface area decreased as the shape approached to a sphere. The total edge length, on the other hand, increased as the cutting length increased and became the maximum for the cuboctahedron. For $c>0.5$, the edge length decreased as $c$ increased and became the minimum for the octahedron, and this value was larger compared to that of the cube. The surface area continued to decrease even when $c$ exceeded 0.5 , and the minimum appeared at $c \cong 0.6$. Then, the value increased and reached a maximum at $c=1$ for the octahedron, which was smaller than for the cube. It should be noted that the orientation of the faces and edges are not included in this figure.

The surface energy is dominated by both the surface area and edge length as 

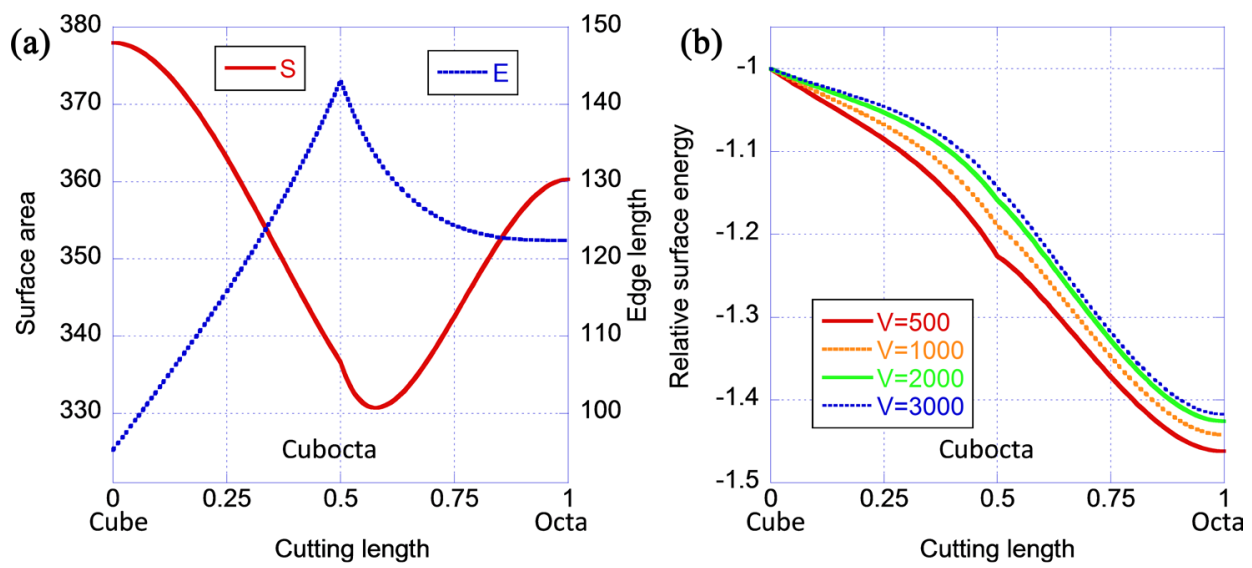

Figure 7. Calculation results based on continuously varied parameters under a constant volume. The cutting lengths $c=0$ and 1 correspond to cube and octahedron, respectively, and the intermediate values represent truncated hexahedron $(0<c<0.5)$, cuboctahedron $(c=0.5)$, and truncated octahedron $(0.5<c<1.0)$. (a) Surface area and edge lendgth; (b) Surface energy.

well as by the crystallographic orientation of the faces and edges. Surface energies were calculated for various volumes and plotted in Figure 7(b), where the value is standardized by the energy for the cube for each volume. The surface energy monotonically decreases as the value in $c$ increases, with a slight discontinuity in the gradient at $c=0.5$ for all volumes. This result shows that the octahedron is, in terms of energy, the most preferable shape. The slopes of the curves are steeper for smaller volumes. This means that a change in shape has a larger impact on smaller particles. Here, it should be noted that the effect of the vertex was excluded in this calculation. In reality, the sharp vertices raise the total energy, leading to instability in the sharp shape. The fair inclusion of the effects is a problem to be addressed in the next stage of our study.

\subsection{Prediction of Surface Energy for Different Models}

In the previous sections, the parameters obtained by MD simulation were used, and they were based on the Lennard-Jones potential, for which the fcc structure and the (111) close-packed plane are stable. In this section, the applicability of the present method to different material systems is verified. The parameters of the constituent faces and edges were virtually varied, and the surface energy of the polyhedral particles was calculated. The parameters of the surface energy for the (100) and (111) faces and (111)-(111) and (100)-(100) edges were virtually varied. The calculated surface energies for $V=500$ and 3000 are shown in Figure 8(a) and Figure 8(b), respectively. In these figures, "base" represents the plot based on the MD results and is identical to the plots in Figure 7(b). In the calculation for " $S_{\text {inv }}$ ", the energies for the (111) and (100) faces were replaced so that the (100) plane has a smaller value than the (111) plane. Similarly, the energies for the (111)-(111) and (100)-(100) edges were replaced for " $E_{\text {inv }}$ ". The overall slope of the surface energy became smaller, but the energy for the cube and octahedron was not reversed by these operations. Therefore, rather exaggerated 

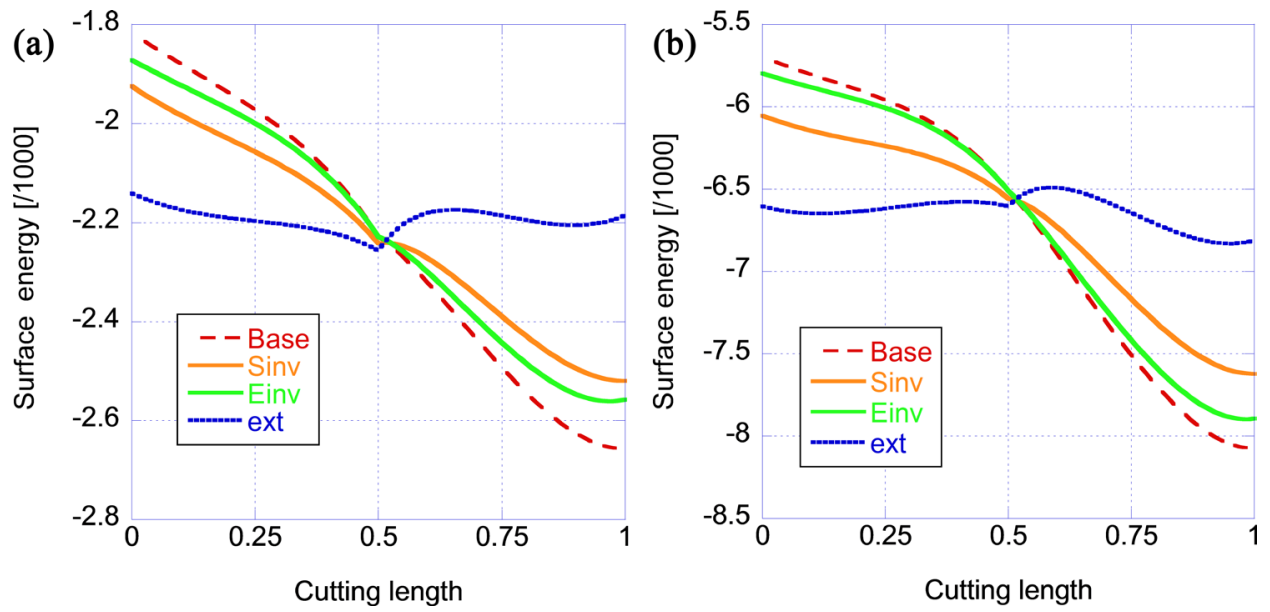

Figure 8. Surface energy vs. cutting parameter for virtualy varied parameters under constant volumes. (a) $V=500$; (b) $V=3000$.

values were provided, and the results are shown by the curves noted as "ext". As a result, the surface energy decreased for the cube and increased for the octahedron, and the values for both shapes reach mostly equivalent values. This means that the stability of various shapes can be explained by providing individual face and edge energies, and it is concluded that the applicability of the proposed model to various material particles is presented.

\section{Conclusion}

In this study, the surface energy of nanoparticles with polyhedral shape was calculated by the MD method and formulated as a polynomial equation. In the MD simulation, a cube and an octahedron were introduced as reference shapes, and various polyhedrons were created by cutting out the vertices. Owing to the stability of the (111) plane, octahedron showed the lowest energy, and the cube had the highest because of the relatively higher energy of the (100) plane. This tendency was fairly captured by the proposed polynomial equation by applying atomic energy despite of a slightly inevitable underestimation. The influence across a few atomic layers should be taken into account for a more precise evaluation. To extend the application range of the proposed equation, the parameters were revised to continuous numbers. The calculated results indicated a proper tendency for fcc particle. Additionally, it was shown that a stability of cubic particles could be obtained by imposing rather exaggerated parameters. This result indicates the possibility of the proposed method being applied to a broad range of materials.

\section{Conflicts of Interest}

The authors declare no conflicts of interest regarding the publication of this paper.

\section{References}

[1] Soares, D.C.F., Domingues, S.C., Viana, D.B. and Tebaldi, M.L. (2020) Poly- 
mer-Hybrid Nanoparticles: Current Advances in Biomedical Applications. Biomedicine \& Pharmacotherapy, 131, Article ID: 110695. https://doi.org/10.1016/j.biopha.2020.110695

[2] Quinson, J. and Jensen, K.M.O. (2020) From Platinum Atoms in Molecules to Colloidal Nanoparticles: A Review on Reduction, Nucleation and Growth Mechanisms. Advances in Colloid and Interface Science, 286, Article ID: 102300. https://doi.org/10.1016/j.cis.2020.102300

[3] Rappaport, D.C. (1995) The Art of Molecular Dynamics. Cambridge University Press, Cambridge.

[4] Long, N.V., Asaka, T., Matsubara, T. and Nogami, M. (2011) Shape-Controlled Synthesis of Pt-Pd Core-Shell Nanoparticles Exhibiting Polyhedral Morphologies by Modified Polyol Method. Acta Materialia, 59, 2901-2907. https://doi.org/10.1016/j.actamat.2011.01.033

[5] Aissa, M.A.B., Tremblay, B., Andrieux-Ledier, A., Maisonhaute, E. Raouafia, N. and Courty, A. (2015) Copper Nanoparticles of Well-Controlled Size and Shape: A New Advance in Synthesis and Self-Organization. Nanoscale, 7, 3189-3195. https://doi.org/10.1039/C4NR06893A

[6] Onaka, S. (2016) Extended Superspheres for Shape Approximation of Near Polyhedral Nanoparticles and a Measure of the Degree of Polyhedrality. Nanomaterials, 6, 27. https://doi.org/10.3390/nano6020027

[7] Yang, L., Feng, J., Ding, Y., Bian, J.J. and Wang, G.F. (2016) An Analytical Description for the Elastic Compression of Metallic Polyhedral Nanoparticles. AIP Advances, 6, Article ID: 085113. https://doi.org/10.1063/1.4961638

[8] Teich, E.G., van Anders, G., Klotsa, D., Dsemuchadse, J. and Glotzer, S.C. (2016) Clusters of Polyhedra in Spherical Confinement. Proceedings of National Academy of Sciences of United States of America, 113, E669-E678.

https://doi.org/10.1073/pnas.1524875113

[9] Lee, Y.H., Lay, C.L., Shi, W., Lee, H.K., Yang, Y., Li, S. and Ling, X.Y. (2018) Creating Two Self-Assembly Micro-Environments to Achieve Supercrystals with Dual Structures Using Polyhedral Nanoparticles. Nature Communications, 9, Article No. 2769. https://doi.org/10.1038/s41467-018-05102-x

[10] Provatas, N. and Elder, K. (2010) Phase-Field Methods in Materials Science and Engineering. Wiley-VCH, Weinheim. https://doi.org/10.1002/9783527631520

[11] Bulent Biner, S. (2017) Programming Phase-Field Modeling. Springer, Cham. https://doi.org/10.1007/978-3-319-41196-5

[12] Uehara, T. (2018) Modeling and Simulation of Particle-Packing Structures and Their Stability Using the Distinct Element Method. Open Journal of Modelling and Simulation, 6, 59-70. https://doi.org/10.4236/ojmsi.2018.64005

[13] Uehara, T. (2015) Phase-Field Modeling for the Three-Dimensional Space-Filling Structure of Metal Foam Materials. Open Journal of Modelling and Simulation, 3, 120-125. http://dx.doi.org/10.4236/ojmsi.2015.33013

[14] Fujiwara, J. (2020) Molecular Dynamics Simulation of Formation and Stability of Metallic Nanoparticles (In Japanese). Master Thesis, Yamagata University, Yonezawa, Japan.

[15] Honeycutt, J.D. and Andersen, H.C. (1987) Molecular Dynamics Study of Melting and Freezing of Small Lennard-Jones Clusters. Journal of Physical Chemistry, 91, 4950-4963. https://doi.org/10.1021/j100303a014

[16] Wang, L., Zhang, Y.N., Bian, X.F. and Chen, Y. (2003) Melting of Cu Nanoclusters by Molecular Dynamics Simulation. Physics Letters A, 310, 197-202. https://doi.org/10.1016/S0375-9601(03)00263-9 